\title{
Análisis de la campaña "La seguridad es tu responsabilidad" impartida por 3M en el incremento de Ventas de EPP en el sector Industrial de Durán en el periodo 2015-2016
}

\section{Analysis of the campaign "Safety is your responsibility" given by $3 \mathrm{M}$ in the increase of EPP sales in the Industrial sector of Durán in the period 2015-2016}

Tatiana S. Macías Muentes

Universidad de Especialidades Espíritu Santo, Ecuador

Juan Diego Pino Maldonado

Universidad de Especialidades Espíritu Santo, Ecuador

Autor para correspondencia: tmacias@uees.edu.ec; juandiegopino1994@gmail.com Fecha de recepción: 7 de Septiembre de 2017 - Fecha de aceptación: 15 de Noviembre de 2017

Resumen: El presente trabajo de investigación pretende mostrar la relación existente entre la aplicación exitosa de una campaña de Marketing y el incremento en ventas. Para probar la hipótesis se usa como ejemplo la venta de EPP en los clientes de 3M en el sector de Durán donde ha sido implementada la campaña La Seguridad es tu Responsabilidad. Este análisis de tipo funcional y relacional se apoya en entrevistas a distintos productores de la campaña, así como de una encuesta realizada a los usuarios directos: jefes de área, mantenimiento, seguridad industrial o seguridad física. La finalidad del estudio es conocer su percepción sobre la campaña y el resultado muestra cómo el uso del Marketing como herramienta en las ventas, genera un efecto positivo en las mismas. Finalmente se recomienda tácticas como incursionar con nuevo material, o esparcir la campaña a otros sectores con el propósito de replicar el éxito obtenido.

Palabras clave: marketing; ventas; epp; campaña; accidentes laborales; $3 \mathrm{~m}$

\begin{abstract}
This research aims to show the relationship between the successful implementation of a Marketing campaign and the increase in sales. To test the hypothesis the sale of EPP in the customers of $3 \mathrm{M}$ in the sector of Durán where the campaign has been implemented Security is your responsibility has been used as an example. This functional and relational type analysis is supported by interviews with different co-workers of the campaign, as well as a survey of direct users: area managers, maintenance, industrial security or physical security. The purpose of the study is to know their perception about the campaign. The result shows how the use of Marketing as a tool in sales, generates a positive effect on them. Finally, we recommend tactics such as penetrating with new material, or spreading the campaign to other sectors in order to replicate the success obtained.
\end{abstract}

Key words: marketing; sales; epp; campaign; work accident; 3m 


\section{Introducción}

La Seguridad Industrial ha tomado fuerza en Ecuador gracias a la implementación de reglamentos técnicos nacionales o de normativas locales reguladas por el INEN para resguardar y proteger los derechos de los trabajadores en la Industria (IEES, Seguridad y Salud en el Trabajo, 2011). Sin embargo, existen empresas en Ecuador que aún no toman conciencia de la necesidad de implementos de protección personal en sus filas de trabajadores ya que probablemente han obviado la relevancia de lo mencionado, alegando una necesidad de reducción de costos o por considerarlo un gasto innecesario. A pesar del instinto de defensa y protección intrínseco en el ser humano, los factores mencionados, derivan en falta de resguardo en la integridad física en los trabajadores y por ende accidentes. (NEUBERG, 2011)

Según el diario el Comercio, en el 2014, 42 de cada 1000 trabajadores en el país sufrieron accidentes laborales y el $40 \%$ de estos fueron catalogados como crónicos. (Torres, 2015). Estos accidentes y distintas circunstancias han obligado a la mayoría de empresas a implementar un departamento interno de Seguridad Industrial y salud ocupacional donde se creen matrices de trabajo con equipos de protección personal y se direccionen actividades que prevengan accidentes. (LEÓN, 2014)

Existen normas internacionales que buscan resguardar la integridad física de los trabajadores y países, como Estados Unidos, Canadá o Colombia que han desarrollado el deseo de poder incluir el uso de los equipos de protección personal como una rutina de la vida diaria. Hay países como EEUU y Canadá que se rigen a través de normativas voluntarias como ANSI o CSA respectivamente, las mismas que crean guías de procesos y estándares de calidad para la fabricación de los EPP (OSHA, 2010). Por otro lado en EEUU existe una organización llamada OSHA que incluso hace parte de la constitución de ciertas normas que rigen para la fabricación de estos equipos, esta fue fundada por el Presidente Nixon en 1970 (EEUU, 2016) Colombia también expone sus medidas por medio del Ministerio de Salud y Protección Social que empieza desarrollando artículos de la Salud Ocupacional en 1979 y luego de la Seguridad Física en el trabajo en 1990. (SOCIAL, 2017). España ya ha empezado a implementar conceptos básicos de la Seguridad Industrial dentro de su industria con la finalidad de resguardar la seguridad física de sus trabajadores, basándose en las normas CE o EN que hacen referencia a la Comunidad Europea (Salud, 2005).

$3 \mathrm{M}$ por su parte es uno de los principales promotores del uso de equipos de protección personal en Ecuador y sus actividades ya sobrepasan los 20 años en el mercado ecuatoriano. (López, 2017) El presente análisis se enfocará en la campaña La seguridad es tu responsabilidad que fue lanzada en el 2016 con la finalidad de apelar a la consciencia de diversos stakeholders como: trabajadores, sus empleadores y las industrias en general para de ese modo, ser un soporte más para las ventas de EPP. Su objetivo principal según aseveran sus creadores es: "Crear consciencia en los trabajadores sobre el uso de correcto de los equipos de seguridad industrial apelando a los sentimientos".

La campaña se compone principalmente de material visual, esto incluye afiches, vídeos y espejos que muestran la leyenda "Te presento al responsable de tu seguridad" O "Ellos te esperan en casa" fundamentando en el antecedente de que la familia, por lo general, espera al padre en su hogar y que el padre busca llegar sano y salvo a su casa para sentir, escuchar, y 
compartir con su familia, según lo explica el Ing. Jorge Cepeda, Coordinador de Negocios de la empresa 3M en la mayoría de clientes de Durán donde la campaña ha sido implementada.

(Cepeda, 2017)

La campaña empieza a regir de manera completa desde el año 2016, en el que luego de su Lanzamiento en el mes de enero, tuvo mucha acogida gracias a la fuerza de ventas de $3 \mathrm{M}$ y sus distribuidores principales. El Ing. Jorge Cepeda nos explica que la campaña fue presentada al $100 \%$ de los clientes claves, considerados por la compañía 3M, los mismos que representan un $70 \%$ de los clientes en la ciudad de Durán. Según Cepeda, fue parte del plan de negocios del año 2016, introducir esta iniciativa en todos los clientes principales para la compañía durante el primer semestre. Se consideraron 63 cuentas claves, de las cuales 61 aceptaron formar parte de esta iniciativa, obteniendo un 97\% de acogida (Cepeda, 2017). Esta medida busca llegar a los sentimientos del trabajador y se ha convertido en un gran soporte para jefes de área, jefes de seguridad industrial, departamento de salud ocupacional y la empresa en general para incentivar el uso de los Equipos de Protección Personal (EPP) y evitar accidentes laborales. La idea se complementó con material visual y auditivo, combinando videos, afiches con capacitaciones y charlas que recalcan la importancia del uso de los EPP y su cuidado.

Según la percepción de los Importadores de EPP, clientes finales y de Multinacionales en el negocio de la Seguridad Industrial, Ecuador en su intento de impulsar la seguridad física dentro del área de trabajo, ha desarrollado ciertas normativas o reglamentos técnicos que mezclan normativas internacionales que lamentablemente han reflejado no ser compatibles, generando resultados ambiguos y confusos para quienes siguen estas disposiciones (López, 2017). Las empresas que buscan tomar medidas, muchas veces no se ven representadas por estas normas ecuatorianas, más bien investigan qué opciones se ofrecen en la industria a nivel internacional y se ha pretendido aplicarla sin una investigación previa exhaustiva del mercado. Esta confusión se refleja con hechos concretos, por ejemplo, en el 2015, en Ecuador se reportó 32,220 accidentes laborales, de los cuales la ciudad de Guayaquil representa el 53\% de los mismos demostrando que, a pesar de las medidas implementadas, el índice de accidentes laborares en esta ciudad es alto comparado con ciudades con mínimos accidentes reportados, como Ambato por ejemplo, según los registros del área de Riesgos en el Trabajo del IEES (Ojeda, 2015). La organización mundial de trabajo indicó hace quince años, durante el congreso mundial sobre seguridad y salud en el trabajo en Viena, que el alto costo por negligencia se debe a una información inadecuada sobre el manejo de la salud y seguridad laboral, hoy en día mantienen ese comunicado por escrito porque sigue las razones presentadas siguen vigentes. (Trabajo, 2017)

En el año 2014 Ecuador creó el INEN, ente regulatorio de la importación de equipos EPP que determina parámetros guía para las empresas al momento de adquirir un equipo pensando en poder resguardar la seguridad física del usuario final. El Servicio Ecuatoriano de Normalización, es el actual responsable de certificar la calidad los EPP y adaptarlos al reglamento ecuatoriano mas no de certificar su producción o su uso dentro de las empresas (INEN, 2014).

3M tienen el mayor porcentaje de participación en el mercado de EPP dentro de Ecuador (Ríos, 2017). Esta es una de las razones principales, por las cuales 3M busca incentivar el uso de los EPP dentro de las industrias que visita y asesora. 3M se siente responsable de la seguridad de 
los usuarios que laboran en empresas que le han brindado la confianza a la marca para protegerlos, por eso busca siempre sobresalir con sus innovadores equipos y brindad mayor comodidad y eficiencia a los usuarios (López, 2017).

Esta investigación fundamenta su estudio en el desarrollo actual de la Seguridad Industrial en Ecuador, en cuanto a la participación de la misma en el mercado y las normativas o reglamentos que actualmente influyen en la comercialización de EPP. El estudio del efecto que esta campaña, ha tenido en el mercado se determinará gracias al análisis del alcance de la compañía 3M en los clientes de la ciudad de Durán y se comparará la información del área de ventas correspondiente al año 2014 hasta el año 2017. Finalmente se especificarán distintas recomendaciones para mejorar el funcionamiento de la campaña y su propósito.

\section{Revisión de la Literatura}

\section{Seguridad industrial}

La Seguridad Industrial ha estado presente desde mucho antes que se defina. Se puede hacer referencia al antiguo Egipto, que cuidaba de sus esclavos en los trabajos de construcción y buscaba siempre la seguridad de quienes servían a los grandes faraones de la historia (Gallegos, 2012). La Seguridad Industrial siempre ha estado ligada a la Salud Ocupacional, donde también se hace referencia a antiguos imperios que buscaban incluir ambas prácticas para resguardas a quienes laboraran para los grandes líderes. Bajo este concepto se destacan figuras como Galeno que 200 años D.C. se encargaba de estudiar las enfermedades que se suscitaban desde las minas hasta los mismos gladiadores siguiendo el ejemplo del gran Hipócrates, considerados por muchos uno de los padres de la medicina ocupacional que 400 años A.C. empezó con el estudio de las enfermedades en esclavos, sobre todo en minas en Roma. (Gallegos, 2012)

La Seguridad Industrial y la Salud ocupacional no terminan de definirse hasta los años 1800 y 1900 donde en Alemania aparecen los primeros escritos y las primeras instituciones como el primer Instituto de Higiene, fundado en Múnich a finales del siglo XIX. En 1918, esta empieza a tomar gran fuerza, cuando Harvard declara la Seguridad y Salud Ocupacional como una licenciatura. (Gallegos, 2012)

\section{Outbound Marketing}

Outbound Marketing los consumidores se asocian a la marca como resultado de varios intentos tradicionales y pagados que se realizan con mucho esfuerzo. (Ciunci, 2016) Este término hace referencia a toda metodología tradicional de hacer publicidad por parte de una empresa, basada en televisión, afiches, radio y otras herramientas.

La finalidad de esta clase de Marketing es de llegar directamente al cliente y poder desarrollar en el mismo, una identidad y lealtad a la marca, gracias a la constante publicidad. Esta estrategia, gracias a sus procesos y los medios que utiliza, ha predominado el mercado del Marketing durante décadas, a pesar de su alto costo. Sin embargo, hoy en día, se ha visto desplazada por el inbound Marketing, que, al ser una herramienta más económica, llega al consumidor de manera rápida y directa por redes sociales o el uso del internet. Así lo demuestra 
Chris Ciunci, que en su libro, Marketing en las trincheras, explica que a la sociedad no le gusta ser empujada hacia un gusto o sentirse asaltada o acosada por una marca, por lo que hoy en día el outbound Marketing está cediendo espacio ante el inbound Marketing.

Existen otras apreciaciones en cuanto a esta técnica de Marketing. En el libro llamado "Cómo Mejorar el inbound y outbound Marketing gracias a una vista confiable del cliente", los autores explican como el inbound Marketing y el outbound Marketing pueden trabajar de la mano para crear confianza en el cliente, sin embargo, fuese imposible conseguir este objetivo sin el impacto que causa el Marketing directo a través del outbound Marketing. (Chuck Ballard, 2015)

Esta técnica de Marketing es la que 3M emplea para poder llegar de manera directa a sus clientes y generar el impacto que desea dentro de la industria, específicamente en el consumidor y no en el comprador. Gracias a esta herramienta, 3M, logra sumergirse dentro de distintas empresas con material visual, el mismo que busca apelar a los sentimientos del trabajador que participa como usuario final y que ve a $3 \mathrm{M}$, como la marca que lo cuida y busca salvar su integridad.

\section{M en Ecuador}

Según un estudio de mercado realizado por $3 \mathrm{M}^{1}$ a inicios del año 2017 , la domina el mercado ecuatoriano de la Seguridad Industrial de manera imponente sobre cualquier otro competidor. En este estudio se mencionan 11 competidores de $3 \mathrm{M}$ y los respectivos porcentajes de dominio en el mercado, haciendo una evaluación de todas las líneas. Este estudio da resultados, considerando los siguientes inconvenientes que merman en el mercado como la presencia de productos falsos, la reducción de productos disponibles para el consumidor por restricciones en importaciones, la recesión económica que afecta en la liquidez del consumidor y finalmente las regulaciones y normativas que rigen por orden del INEN al momento de poder trabajar con un EPP.

$3 \mathrm{M}$ ha palpado un gran avance en su participación de mercado pasando del $42 \%$ de dominio en el 2014, a un 57\% de dominio en el 2016. Un crecimiento exponencial que se debe mucho a las nuevas adquisiciones de la marca como es el caso de la compra de Capital Safety Corp. Este crecimiento refleja un dominio absoluto de la marca, con el 51\% de participación en el mercado, seguido por su principal competidor, la multinacional, Kimberly Clark. (Ríos, 2017)

Este crecimiento no solo ha significado un aumento en ventas, sino que también les ha arrebatado espacio a sus competidores. Así lo refleja el decrecimiento de Kimberly Clark, que en el 2015 representaba un 12\% de presencia en el mercado y en el 2016 reduce su participación en un 50\% (Ríos, 2017). Este estudio demuestra que una de las competencias más fuertes en el mercado de la Seguridad Industrial para 3M, es la falsificación de sus productos, estos tienen un $5 \%$ de participación en el mercado (Ríos, 2017). Este inconveniente se ha convertido en un reto

\footnotetext{
1 3M: Minnesota, Minning and Manufacturing company, es una Multinacional estadounidense dedicada a investigar, crear, desarrollar y comercializar equipos en distintas líneas de trabajo como la medicina, tecnología y de más.
} 
para la fuerza de ventas de la compañía que ha tenido que incrementar esfuerzos con mayor énfasis en campañas masivas que denuncien el delito. Gracias a este inconveniente se ha abierto la posibilidad de acompañar las ventas con distintas ideas, una de estas la campaña de la Seguridad es tu Responsabilidad que apela mucho a la importancia de los EPP en la vida del trabajador.

\section{La seguridad es tu responsabilidad}

Esta campaña lanzada por 3M en el 2016 se concibe años anteriores, precisamente en la gestión del Ing. Roberto Nowak cuando era el gerente de Marketing de la multinacional en el Ecuador. Fue en el año 2009 cuando la idea empezó a germinar no como campaña, sino con un servicio post venta que incluía charlas que apelaban al sentimentalismo de los trabajadores. (Nowak, 2017). En el 2013 se empiezan a realizar levantamientos de información gratuitas a clientes con la finalidad de sugerir EPP fabricados por 3M en las distintas empresas. Nowak explica que esta campaña nace como una respuesta a la baja reposición de EPP en el mercado y que justamente el objetivo de incrementar las ventas, debía verse acompañado de medidas distintas que rompan con lo tradicional del mercado, donde los servicios post ventas se resumían en capacitaciones sobre el uso y cuidado de los equipos para alargar su tiempo de vida útil. (Nowak, 2017)

La campaña va tomando forma en el 2012 cuando se empieza a transmitir en los clientes, vídeos del slogan global para la línea de $S \& G$, El poder de proteger tu mundo. De ahí nace la idea de implementar material visual, con el Ing. Tommy Pulley heredando un proyecto. En el 2014 3M empieza a trabajar en el vídeo y la campaña empieza a tomar forma. Es así como en este mismo año se define el nombre de la Seguridad es tu Responsabilidad, y la empresa empieza a recopilar todo lo que pueda sumar al lanzamiento de una campaña local donde las empresas sean las principales promotoras. Es así como Pulley lanza en el 2015 la campaña de la Seguridad es tu Responsabilidad respaldada por afiches, un vídeo, un espejo como la principal novedad mostrando la leyenda "te presento al responsable de tu seguridad" y una gama de servicios post venta que incluyen, levantamiento de riesgos y matrices más un informe técnico, capacitaciones al personal sobre el uso y cuidado de los equipos de Seguridad y el acompañamiento en ferias dentro de las industrias para promulgar el uso de los EPP dentro de las actividades laborales del usuario. (Pulley, 2017)

La campaña ha sido implementada en más de 300 empresas a nivel nacional, teniendo una mejor aceptación en las empresas ubicadas en Guayaquil y Durán. La misma sigue vigente y hasta la fecha no existe algún cambio o complemento y sus resultados siguen siendo evaluados en la actualidad. Esta campaña busca apelar a la sensibilidad del trabajador, tratando de rescatar a la familia como prioridad y demostrando como un individuo siempre es esperado en casa por sus seres queridos, esto con la finalidad de que el usuario final, demande el uso de los equipos e implementen una cultura de trabajo seguro en su entorno.

\section{Metodología}

Tipo de estudio 
La investigación que se desempeña combina la metodología cualitativa y la cuantitativa, dividiendo el documento en dos partes. La primera parte busca resaltar los puntos positivos de la campaña implementada por $3 \mathrm{M}$ y sus consecuencias en la industria ecuatoriana. Para esto se describe la participación de la marca y de la campaña dentro de las distintas empresas del sector de Durán y como está ha afectado en la comercialización de los EPP. La segundase enfoca en un estudio cuantitativo que compara los resultados de la encuesta realizada a diferentes clientes de 3M en el sector de Durán y termina con la comparación de ventas de EPP por parte de 3M en el campo de la Seguridad Industrial, en los dos últimos años. La investigación realizada es de tipo descriptivo y analítico pues proporciona conclusiones y recomendaciones, una vez concluido tanto el análisis como la descripción de los procesos de la campaña y su participación en el mercado. El propósito central es medir el efecto de la campaña con respecto a ventas y con relación a concientizar acerca de la importancia del uso de equipos de protección personal en el campo laboral.

\section{Participantes}

Se considera relevante incluir únicamente a quienes están directamente involucrados en el manejo de EPP dentro de las empresas: los jefes de seguridad industrial y auxiliar de este campo, jefes de mantenimiento, jefes de taller, personal de seguridad física, jefes de producción y auxiliar, encargados de planta o jefes de la misma. La ubicación seleccionada es el área de Durán, sitio donde la campaña ha sido implementada.

\section{Instrumentos}

A lo largo de la investigación se utilizan distintas referencias para poder corroborar la información que se describe. Adicionalmente, se utilizan dos técnicas para llevar a cabo esta investigación, la primera hace referencia a entrevistas realizadas a diversas personas que actualmente laboran en la compañía 3M, y que están involucrados de manera directa con la comercialización de EPP o con la campaña de la Seguridad es tu Responsabilidad. Esto va acompañado de una entrevista a un ex funcionario de la empresa y mentor principal de la propuesta en mención

Por otro lado, se utiliza la técnica de encuesta basada en 10 preguntas a través de las cuales se busca extraer información para evaluar y analizar el efecto de la campaña dentro de las empresas. Estas encuestas son dirigidas únicamente a los participantes previamente señalados.

\section{Análisis de resultados}

Para obtener datos reales y analizar el efecto de la campaña La Seguridad es tu Responsabilidad, se realizó 59 encuestas entre los participantes descritos previamente correspondientes a los diferentes clientes de $3 \mathrm{M}$ en el área de Durán y se obtuvo los siguientes resultados.

\section{1. ¿A qué sector pertenece su empresa?}




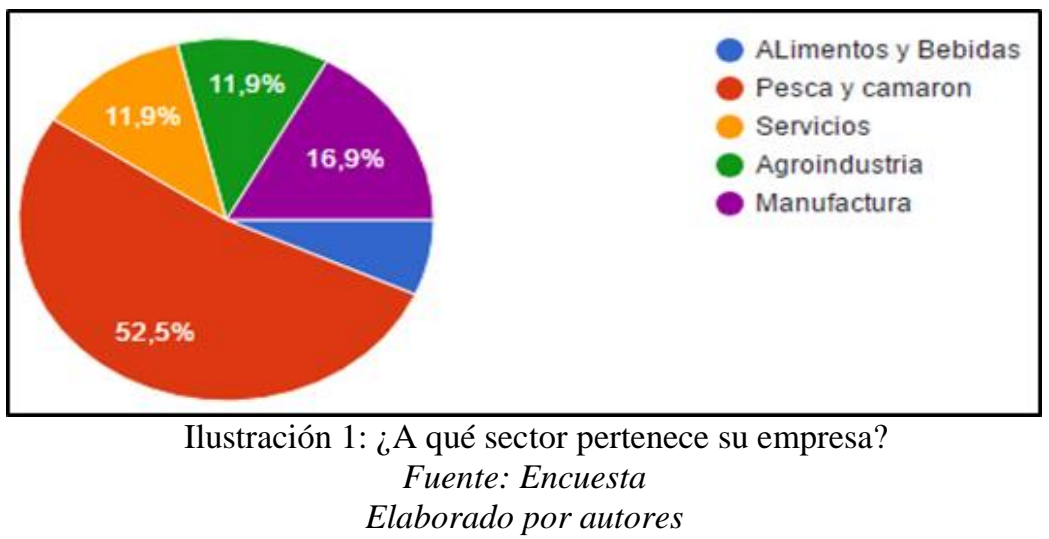

Estas encuestas fueron realizadas a empresas de distintos sectores comerciales donde la campaña ha sido implementada para poder comparar como esta puede ser introducida sin importar la actividad económica que se realice, siempre y cuando, $3 \mathrm{M}$ ya tenga presencia dentro de esta Industria. Los clientes de 3M dentro de Durán y que cumplen con los requisitos de la encuestas, son mayormente del área de pesca y camarón, conformando un 52,5\%, mientras en el otro extremo, las empresas de alimentos y bebidas en esta localidad y que tienen la campaña implementada se dedican a la elaboración de alimentos o bebidas.

\section{2. ¿A qué sector pertenece su empresa?}

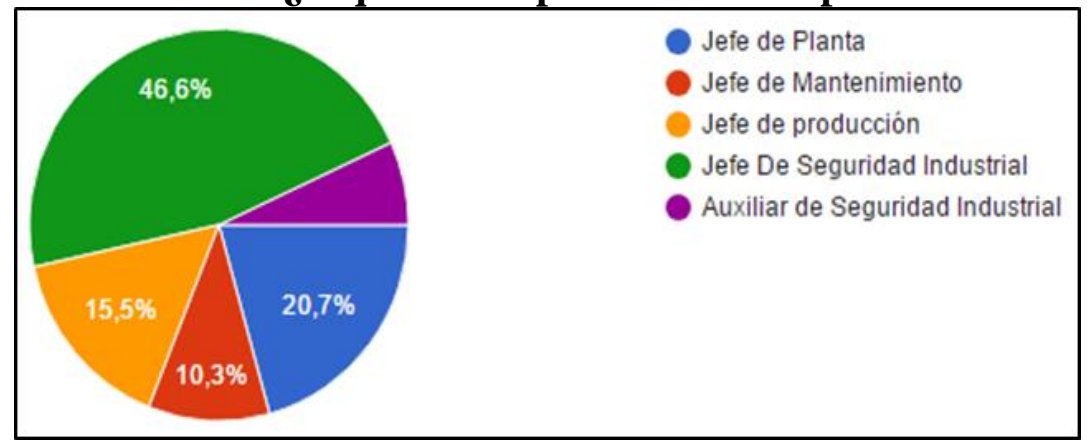

Ilustración 2: Seleccione su cargo dentro de la empresa:

Fuente: Encuesta

Elaborado por autores

Estas encuestas fueron realizadas únicamente a jefes de planta, jefes de mantenimiento, jefes de producción, jefes de seguridad industrial y auxiliares se seguridad industrial pues se consideran a estos con un mayor vínculo con la campaña de y una mayor relación con los ejecutivos de ventas y asesores técnicos de la compañía 3M, para entender los beneficios que se ofrecen. La mayor cantidad porcentual de encuestados pertenece a los jefes de seguridad industrial quienes son los que mayormente se relacionan en el día con la campaña y velan por los interéses de los objetivos de la misma porque son similares a los de ellos. Los Jefes de planta con el 20,7\% también representan una cantidad interesante pues ellos son los responsables de prevenir accidentes laborales en el área a su cargo y que el material visual que se implemente realmente siga los lineamientos propuestos. Los auxiliares de Seguridad Industrial tan solo ocupan el $6.27 \%$ de los encuestados pues la mayoría de empresas encuestadas no cuentan con personal para este cargo. 


\section{Tiempo de la campaña dentro de la empresa}

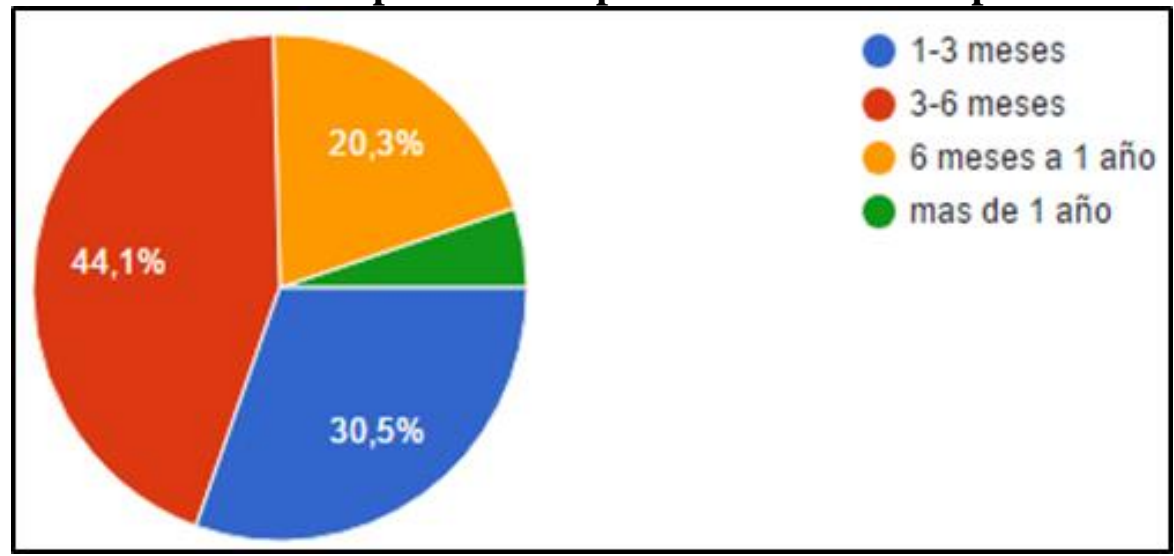

Ilustración 3: Tiempo de la campaña dentro de la empresa

Fuente: Encuesta

Elaborado por autores

La campaña fue lanzada en Enero del 2016. De acuerdo a estas estadísticas, el primer semestre atravesó dificultades para su implementación, pues de las empresas encuestadas tan solo representan el 5,1\%. El esfuerzo por implementar la campaña empezó a dar pequeños pasos de manera más acelarada, por tanto las empresas con la camapaña implementada entre 6 meses y 1 año, ya abarcan el 20,3\%. Una vez que se tomo el hilo a esta actividad, la presencia de la campaña aumentó en el sector de Durán, por lo que el 44,1\% de las empresas encuestadas tienen de 3 a 6 meses la campaña implementada. La misma sigue dando sus frutos hoy en día y muchas de estas empresas terminaron el año con la campaña o han iniciado con la misma. Esta información cuadra con los datos dados por el Coordinador de negocios asignado a la zona Durán, quien en su entrevista explica como el segundo y tercer trimestre del año 2016 fueron los que más empeño se puso a la campaña para que los resultados se den por sentado. (Cepeda, 2017)

\section{4. ¿Cuál fue el elemento más productivo?}

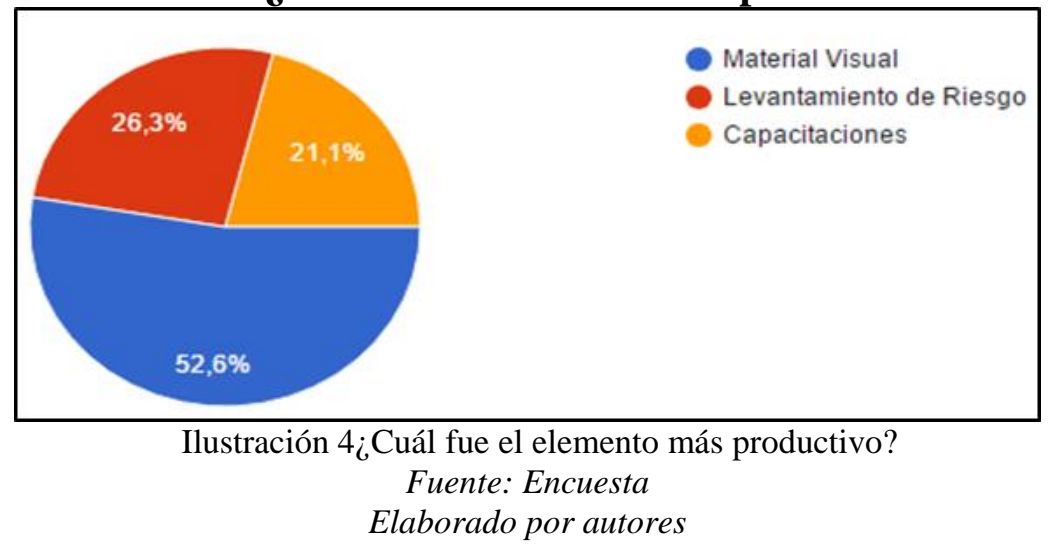

Para los usuarios encuestados que tienen una mayor afinidad con las ventajas de la campaña dentro de la empresa, el material visual es mayormente importante comparado a sus otros dos elementos con el 52,6\% pues de seguro es lo que mayor impacto causa en el usuario.

\section{5. ¿Se han implementado todos los elementos?:}




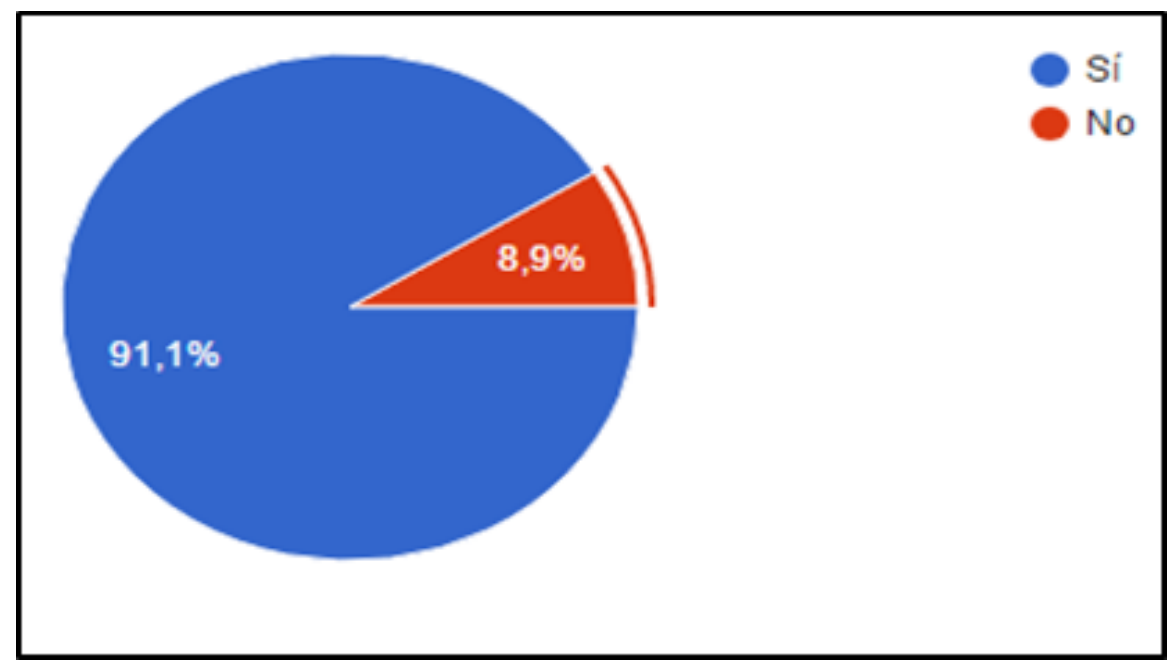

Ilustración 5: ¿Se han implementado todos los elementos?:

Fuente: Encuesta

Elaborado por autores

De las respuestas obtenidas podemos decir que 51 fueron afirmativas mientras 5 afirman no haber recibido todos los elementos de la campaña. Esto indica un gran porcentaje de eficiencia por parte de los asesores y ejecutivos de ventas por introducir de buena manera la campaña dentro de una firma.

\section{Del 1 (muy malo) al 5 (excelente), evalúe el material visua}

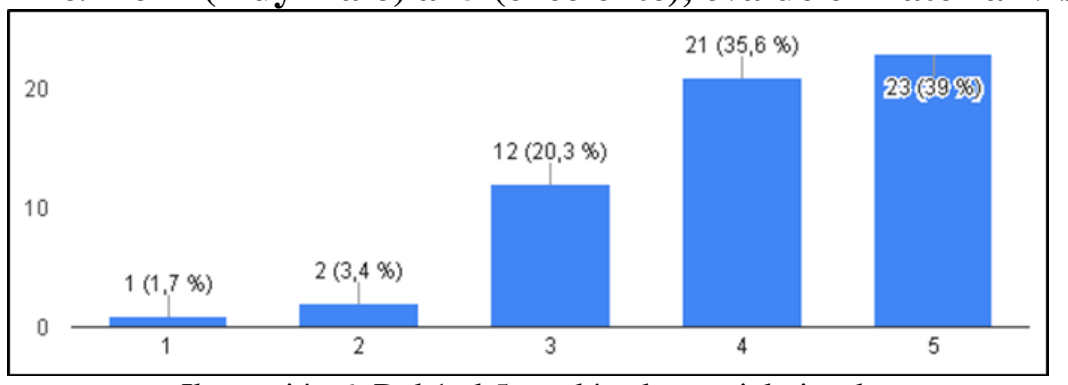

Ilustración 6: Del 1 al 5, evalúe el material visual:

Fuente: Encuesta

Elaborado por autores

En esta pregunta, se le otorga un rango de calificación al material visual donde 1 es muy malo y 5 es excelente. Para la mayoría de los encuestados, la calificación del material va de 4 a 5 , sumando ambos porcentajes, se obtiene un total del 74,6\% de personas que consideran que el material de la campaña es muy bueno o excelente. Tan solo un usuario de los encuestados, considera que el material es muy malo.

7. Del 1 (muy malo) al 5 (excelente), evalúe el material de las capacitaciones: 


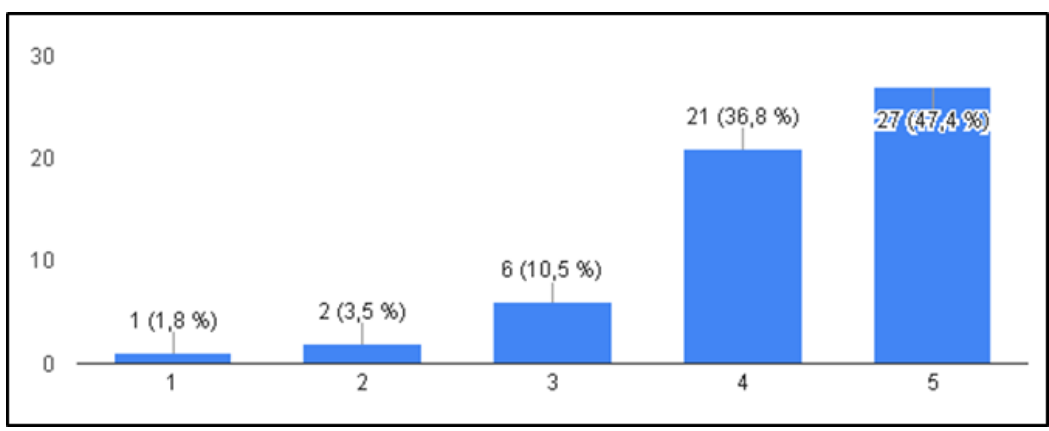

Ilustración 7: Del 1 al 5, evalúe el material de las capacitaciones:

Fuente: Encuesta

Elaborado por autores

El 47,4\% de la muestra considera que las capacitaciones son excelentes, seguidas de 21 personas que representan el $36,8 \%$ que consideran que las capacitaciones son muy buenas. Nuevamente el $\%$, que equivale a un participante, considera que las capacitaciones son muy malas.

\section{Del 1 (muy malo) al 5 (excelente), califique la evaluación de levantamiento de} riesgos

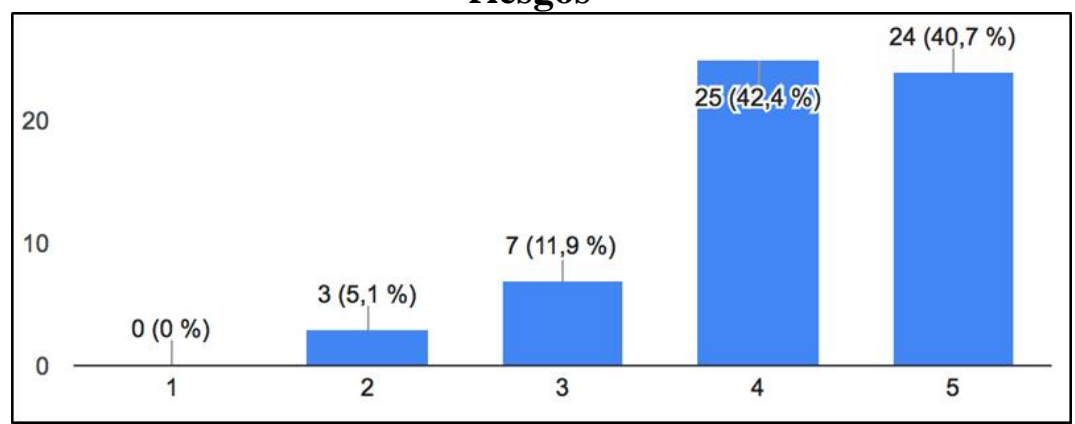

Ilustración 8: Del 1 al 5, califique la evaluación de levantamiento de riesgos

Fuente: Encuesta

Elaborado por autor

La mayoría de los encuestados califican los levantamientos de riesgo de 4 a 5, obteniendo un $83,1 \%$ de los usuarios que consideran que los levantamientos de riesgos son muy buenos o excelentes. Lo curioso en estos resultados se da en la calificación 1 donde no existe usuario que considere que los levantamientos de riesgo son muy malos.

\section{Objetivo principal de la campaña dentro de su empresa:}

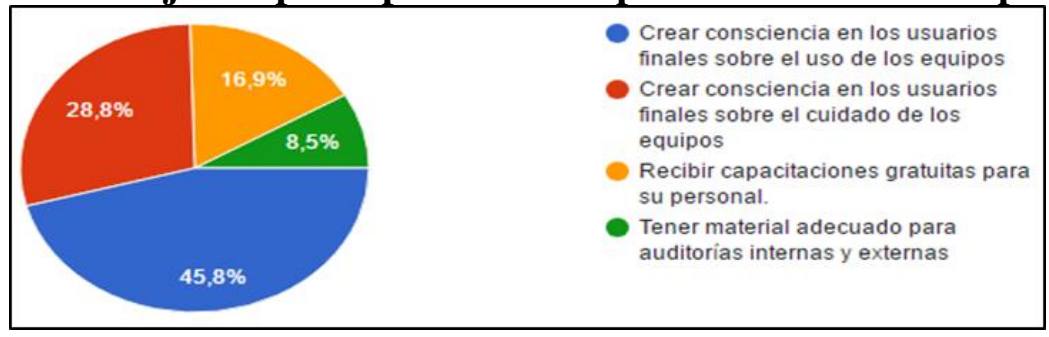

Ilustración 9: Objetivo principal de la campaña dentro de su empresa

Fuente: Encuesta

Elaborado por autores 
La mayoría de usuarios, con un 45,8\%, consideran que han aceptado implementar la campaña dentro de sus empresas para poder crear consciencia en los usuarios finales sobre el uso de los equipos, enalteciendo la importancia de los mismos. Luego con un $28,8 \%$ los usuarios encuestados consideran que la campaña va a ayudar en el cuidado de los equipos con la finalidad de prolongar la vida útil de los mismos.

\section{0. ¿Se cumplió el objetivo que marcó en la pregunta anterior?}

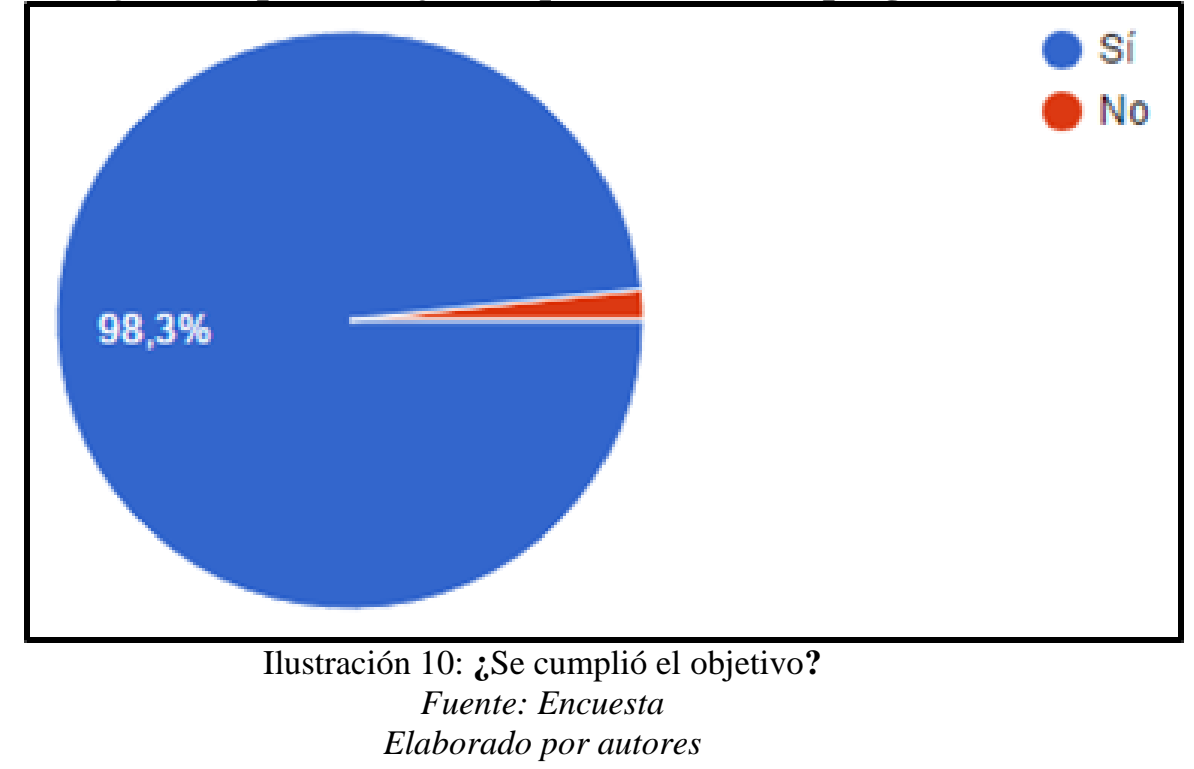

De todos los encuestados, tan solo un usuario considera que implementar la campaña dentro de la empresa no dio los resultados esperados y no cumplió el objetivo que este usuario solicitaba. Este usuario mencionó que accedió a la campaña con el fin de obtener material adecuado para auditorías internas o externas y considera que la campaña no ha cumplido con esa función. El restante de los encuestados considera efectiva la campaña.

\section{Análisis de ventas 2015 vs 2016.}

3M entrega la información de su POS DATA, reporte mensual que registra la venta de sus equipos a través de todos sus distribuidores a nivel país. Durán representa el segundo sector más importante de la región Costa en cuanto ventas se refiere, superando a ciudades como Manta o Machala y siendo tan solo superada por Guayaquil (Cepeda, 2017). De acuerdo al informe, Durán representó un 18\% de las ventas totales de EPP de la compañía en el año 2015, en comparación con Guayaquil que representa el 23\% (CAICEDO, WALTER, 2016). En el 2016 estos números cambian y disminuyen su distancia porcentual, pues según el reporte, Durán pasa a ocupar el $21 \%$ de las ventas totales y Guayaquil tan solo aumenta un punto porcentual, representando el $24 \%$ de las ventas totales. En total de año a año, existe un crecimiento del $26 \%$ en el sector mencionado. (CAICEDO \& RÍOS, 2017). Las ventas van a ser analizadas y comparadas trimestralmente en ambos años.

Primer trimestre 2015 vs primer trimestre 2016 
El primer trimestre del año 2015 registra ventas totales de $\$ 200,504.00$ en el sector de Durán. De este monto, el $62 \%$ de sus ventas se ve reflejado en industrias dedicadas a la pesca y exportación de camarón, tales como Promarisco, Expalsa, Pronaca, Skretting, entre otras. En el año 2016 las ventas aumentan en un 24\%, arrojando un total de ventas de EPP de $\$ 247,632.71$ (CAICEDO, WALTER, 2016).

Segundo trimestre 2015 vs segundo trimestre 2016.

En este segundo trimestre el año 2015, refleja un monto total de ventas de $\$ 218,591.14$ comparado a los $\$ 253,682.69$ del segundo trimestre del año 2016. Esto significa un 16\% de crecimiento en ventas netas de la compañía $3 \mathrm{M}$ en sus clientes del sector de Durán. En este trimestre la campaña tuvo una acogida del 20,3\% de los encuestados, sumada al 5,1\%, significaba que más de un cuarto de los encuestados tenía la campaña implementada en sus distintas plantas (CAICEDO, WALTER, 2016) .

Tercer trimestre 2015 vs tercer trimestre 2016.

El tercer trimestre del año 2015 refleja un total de ventas de EPP de \$215,615.10 En comparación al total de ventas de EPP en el mismo trimestre del año 2016 donde se registra un monto de $\$ 299,354.56$. Este es el crecimiento más grande de los trimestres comparados, pues refleja un 39\% más de ventas. En este mismo trimestre, según las encuestas, el 44,1\% de la población implementó la campaña en este trimestre (CAICEDO, WALTER, 2016).

\section{Cuarto trimestre 2015 vs cuarto trimestre 2016.}

Este trimestre refleja un monto de ventas de EPP de \$211,258.88 en el 2016 el crecimiento del $25 \%$ nos deja un total de ventas de EPP de \$263,043.97. En este trimestre la campaña tuvo acogida en el 30,5\% de la población encuestada (CAICEDO, WALTER, 2016) .

Tabla 1: Tabla Comparativa De Ventas Trimestrales 2015-2016 Durán

\begin{tabular}{|c|c|c|c|c|c|}
\hline \multicolumn{6}{|c|}{ Tabla Comparativa De Ventas Trimestrales 2015-2016 Durán } \\
\hline AÑO & 1 & 2 & 3 & 4 & TOTAL \\
\hline & TRIMESTRE & TRIMESTRE & TRIMESTRE & TRIMESTRE & \\
\hline 2015 & $\$ 200,504.03$ & $\$ 218,591.14$ & $\$ 215,615.06$ & $\$ 211,258.81$ & $\begin{array}{c}\$ \\
84596903\end{array}$ \\
\hline 2016 & $\$ 247,632.73$ & $\$ 253,682.60$ & $\$ 299,354.54$ & $\$ 263,043.90$ & $\begin{array}{c}\$ \\
1,063,713.77\end{array}$ \\
\hline $\begin{array}{c}\text { CRECIMIENT } \\
\text { O }\end{array}$ & $24 \%$ & $16 \%$ & $39 \%$ & $25 \%$ & $26 \%$ \\
\hline
\end{tabular}

Fuente: Pos Data 3M.

Elaborado Por Autores 


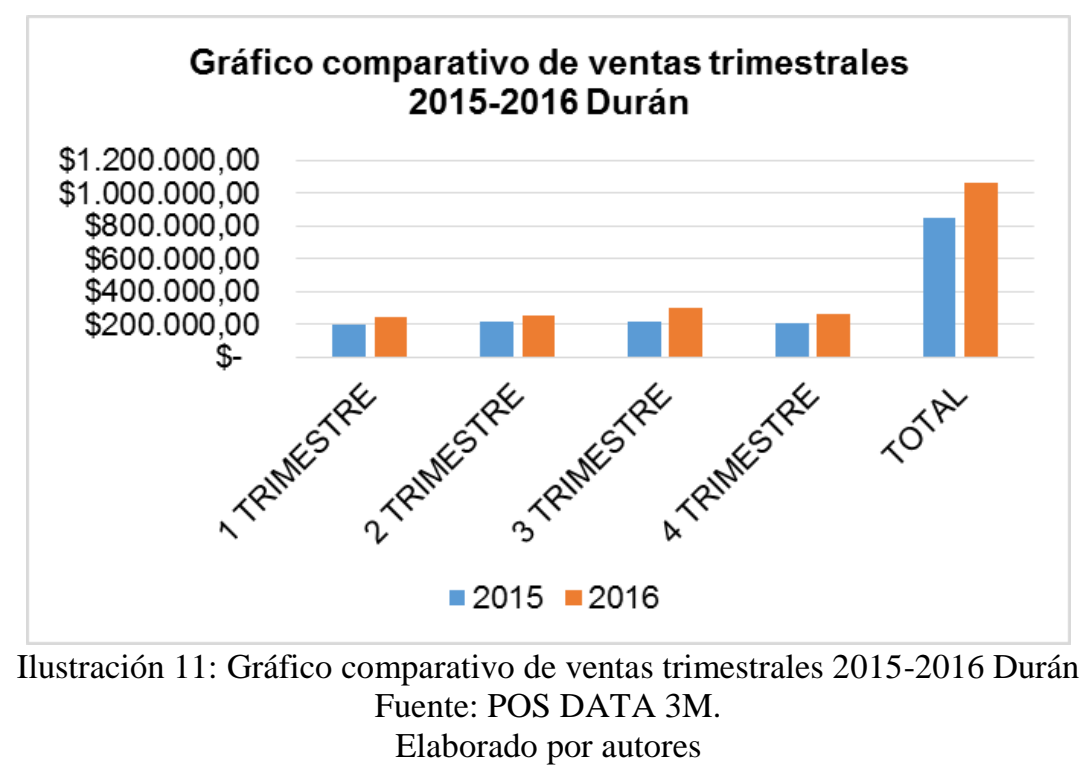

\section{Conclusiones}

A lo largo de la investigación se pretende entablar una relación entre la campaña de Marketing de 3M, La Seguridad es tu Responsabilidad, y la presencia de la marca con los EPP dentro de las compañías, por ende, el incremento de ventas por parte de los distribuidores autorizados dentro de la industria ecuatoriana. Para evaluar la hipótesis de que dicha campaña tiene un efecto positivo en las ventas de EPP en el sector de Durán se realizó encuestas, cuyos resultados permitieron comprobar que los clientes aceptan favorablemente y, por tanto, se alcanzan los objetivos planteados por la campaña. Además, este resultado permitió evaluar el material que se utiliza en la campaña y la aceptación que la misma ha tenido en el mercado de Durán.

Por otro lado, 3M proporcionó su POS DATA, que son registros de ventas de EPP en los clientes de Durán a través de todos sus distribuidores, permitiendo a la investigación conocer a ciencia cierta los valores porcentuales de ventas por trimestre y el cambio de un año al siguiente. Con esta información se puede corroborar más la teoría, gracias a sus valores netos en dólares de las ventas. Esta nos permite comparar el alcance de ventas en las cuentas claves donde ha sido implementada la campaña para poder evaluar el impacto que tuvo la misma en el incremento de las ventas.

Gracias a las encuestas realizadas, podemos observar el compromiso de $3 \mathrm{M}$ con su campaña y sus clientes, pues han logrado abarcar a la mayoría de los clientes del sector en el transcurso de un año calendario. Así también podemos evaluar el deseo de los empleadores de comprometerse con la Seguridad Industrial dentro de sus plantas, al aceptar tener la campaña para cumplir con un fin. Para corroborar esto, se observa la novena pregunta de la encuesta, que indica que el $45.8 \%$ de los encuestados desea tener la campaña para poder crear conciencia en sus trabajadores sobre la importancia de utilizar un EPP a la hora de realizar sus actividades laborales, tratando de resguardar riesgos y prevenir accidentes. 
La investigación planea evaluar la campaña y sus servicios con distintas preguntas en las encuestas. Entre éstas se observa cómo la mayoría se inclina por el material visual como el eje principal de la campaña dentro de la empresa, corroborando el objetivo de $3 \mathrm{M}$ de poder causar impacto a través de material visual que apele a los sentimientos del individuo que trabaja y de esa manera crear conciencia sobre la importancia del uso de un EPP al momento de trabajar. Entre los encuestados, el 39\% califica el material visual como excelente, mientras que la siguiente categoría "muy bueno", fue seleccionado por más del 35\%. Esto permite concluir que la elaboración del material visual de la campaña ha sido acertada ya que logra cumplir la función que la marca quiere con esta herramienta. Por otro lado, las capacitaciones también ocupan un rol importante dentro de los objetivos de los usuarios ligados directamente con la Seguridad Industrial dentro de una empresa.

El 28,8\% de encuestados consideran que el objetivo principal de la campaña dentro de sus lugares de trabajo consiste en crear conciencia sobre el uso y cuidado de los EPP y es así que esta meta se ve atendida con las capacitaciones impartidas dentro de la operación. Por otro lado, el 16,8\% de encuestados considera que la función principal de la campaña es tener capacitaciones gratuitas para su personal, corroborando que el incluir estos entrenamientos ha sido un gran acierto para la campaña. La encuesta muestra cómo solo una persona no está satisfecha con el contenido de las capacitaciones, mientras más del $47 \%$ de los encuestados considera que el material utilizado en las capacitaciones es excelente.

Gracias a estos resultados se puede concluir que la combinación de tres recursos distintos se ha complementado de manera excelente, teniendo gran acogida por parte de las distintas plantas en Durán.

Como factor final a considerar para determinar que la campaña realmente ha influenciado en las ventas de EPP de manera positiva en el 2016 (en comparación al 2015 donde no había sido lanzada aún) se analiza el aumento de ventas de un año al otro. El incremento final es del 26\%, un aumento de bastante importancia, pues la meta de 3M de crecimiento anual era del $20 \%$ (Ríos, 2017). Se debe considerar que la mayoría de clientes aceptó implementar la campaña de La Seguridad es tu Responsabilidad, en el periodo de un año, brindando un alto porcentaje de aceptación en el mercado.

Estos datos confirman la hipótesis descrita en la presente investigación. La campaña de Marketing que 3M implementó ha sido un factor determinante para poder sobrepasar expectativas y metas de crecimiento al igual que afianzarse en un mercado altamente competitivo. La zona donde se llevó a cabo la investigación tuvo una respuesta positiva ante el experimento de la campaña, lo cual abre la posibilidad de poder replicar esta estrategia en distintas zonas.

\section{Recomendaciones}

Por el resultado arrojado en la última pregunta de la encuesta se permite recomendar lo siguiente: Entre la mayoría de las respuestas que se recogieron en esta investigación, los usuarios recomiendan: 
Primero, incremento de material visual y alineación con el/los logo(s) de la compañía. Por tanto, se recomienda a la empresa, entregar el arte original del material visual, por ejemplo, afiches o diseño del espejo, así como los permisos necesarios para que las distintas firmas que gozan de la campaña puedan reproducirlos directamente y/o modificarlos de modo que se pueda personalizar, por ejemplo, incluyendo su logo junto al de 3M. De esta manera los usuarios finales de las distintas plantas se van a sentir más identificados con sus empleadores y $3 \mathrm{M}$, creando así lealtad a la marca tanto de los usuarios como de los jefes involucrados con la Seguridad Industrial. Además, se podrá crear reconocimiento de marca, gracias al aumento de impresiones que van a ser colocadas en lugares estratégicos de las empresas.

Segundo, inclusión de un evento masivo anual que una a todas las empresas que gozan de la campaña con el fin de compartir las mejores prácticas y casos de éxito. Por ende, se recomienda a la multinacional, crear foros de discusión y compartir experiencias con empresas relacionadas para que puedan compartir experiencias y opiniones que van a servir de retroalimentación para 3M. Además, se va a lograr generar matrices similares con sus equipos, dentro de varias compañías, generando un dominio mayor dentro del mercado.

Tercero, crear una alianza de la compañía 3M con un instituto educativo de nivel superior. Así se va a poder validar sus capacitaciones con certificados personales para cada usuario que recibe esta instrucción. Gracias a esto, se generará mayor formalidad en su trabajo, además de ser una carta de entrada para educar a los usuarios en el uso, cuidado e importancia de los equipos.

\section{Bibliografía}

CAICEDO, W., \& RÍOS, C. (2017). Post Data 2016. Ecuador: 3m.

CAICEDO, WALTER. (2016). Pos Data. Ecuador: 3m.

Cepeda, J. (24 de Marzo de 2017). La campaña dentro de las cuentas claves en Durán. (J. D. Pino, Entrevistador)

Chuck Ballard, J. C. (2015). Enhance Inbound and Outbound Marketing with a Trusted Single View of the Customer. IBM Books.

Ciunci, C. (2016). Marketing in the trenches. Lulu publishing services.

D.S., A. (17 de NOVIEMBRE de 2005). Seguridad privada :si consideramos que el hombre, dejo de ser nómada. Obtenido de Seguridad privada :si consideramos que el hombre, dejo de ser nómada.: http://search.proquest.com/docview/311233436?accountid=36753

EEUU, D. d. (2016). Todo sobre la OSHA. Estados Unidos.

Gallegos, W. L. (2012). Revisión Histórica De La Salud Ocupacional Y La Seguridad Industrial. Revista Cubana de Saluds y Trabajo, 8. 
IEES. (2011). Revista Técnica Informativa del Seguro General de Riesgos del Trabajo. Seguridad y Salud en el trabajo.

IEES. (2011). Seguridad y Salud en el Trabajo. Revista Técnica Informativa del Seguro General de Riesgos del Trabajo.

INEN. (2014). Instituto De Normalizacion Ecuatoriana. Recuperado El 27 De Febrero de 2017, de http://www.normalizacion.gob.ec/la-institucion/

LANDAZURI, L. J. (2015). Estudio De Factibilidad Para La Creación De Una Empresa Importadora Y Comercializadora De Equipos De Protección Personal Para El Sector De La Construcción En La Ciudad De Quito. Quito, Pichincha, Ecuador.

LEÓN, M. G. (2014). Informe de Rendicion de Cuentas 2014.

López, I. R. (4 de Abril de 2017). La Seguridad Industrial desde una perspectiva técnica. (J. D. Pino, Entrevistador)

NEUBERG, S. (2011). Human threat management system: Self-protection and disease avoidance. Neuroscience and Biobehavioral Reviews, 1042-1051.

Nowak, R. (17 de MARZO de 2017). 3m La Seguridad Es Tu Responsabilidad 1 Parte. (J. D. PINO, Entrevistador)

Ojeda, I. P. (2015). Rendición de Cuentas 2015.

Organizacion Mundial del Trabajo. (2016). Recuperado el 09 de septiembre de 2016, de http://www.ilo.org/global/about-the-ilo/newsroom/news/WCMS_071435/lang-es/index.htm

OSHA. (2010). Hoja de Datos OSHA. E.E.U.U.

Pulley, I. T. (15 de Marzo de 2017). Las bases de la campaña. (J. D. Pino, Entrevistador)

Ríos, C. (2017). S\&G PLAN 2017. Ecuador: 3M.

Salud, O. M. (2005). El número de accidentes y enfermedades relacionados con el trabajo sigue aumentando. Obtenido de Organización Mundial de Salud: http://www.who.int/mediacentre/news/releases/2005/pr18/es/

SOCIAL, M. D. (6 de Abril de 2017). MINSALUD. Obtenido de MINSALUD: https://www.minsalud.gov.co/Paginas/Norm_Leyes.aspx\#InplviewHash8124f488-25304037-9cc8-da5150e170f5=Paged\%3DTRUE-p_GroupCol1\%3D1988p_GroupCol2\%3DPensiones-PageFirstRow\%3D31-WebPartID\%3D\%7B8124F488-2530--4037--9CC8--DA5150E170F5\%7D

Torres, A. (15 de Mayo de 2015). El Comercio. Obtenido de El Comercio: http://www.elcomercio.com/actualidad/trabajadores-accidenteslaborales-iessempresas.html 
Trabajo, O. I. (2017). Organización Internacional del Trabajo. Obtenido de http://www.ilo.org/global/about-the-ilo/newsroom/news/WCMS_071435/lang-es/index.htm 